\title{
DEFORMATION QUANTIZATION OF KÄHLER MANIFOLDS
}

\author{
NIKOLAI RESHETIKHIN AND LEON A. TAKHTAJAN
}

\author{
AbStRact. We present an explicit formula for the deformation quantization \\ on Kähler manifolds. \\ Dedicated to our teacher Professor L.D. Faddeev on his 65th birthday
}

\section{INTRODUCTION}

The concept of quantization has a long history. Mathematically, it originated from Hermann Weyl [1], who introduced a mapping from classical observables functions on the phase space $\mathbb{R}^{2 n}$ - to quantum observables - operators in the Hilbert space $L^{2}\left(\mathbb{R}^{n}\right)$. The idea was to express functions on $\mathbb{R}^{2 n}$ as Fourier transforms and to replace the exponential kernel - the one-dimensional representation of the Heisenberg group - by the corresponding unitary group element in the von Neumann infinite-dimensional representation of the Heisenberg group, parameterized by the Planck constant $\hbar$. The inverse mapping was constructed by E. Winger 22 by interpreting classical observables as symbols of operators. Later on, Moyal [3] and Groenewold [4] have shown that the symbol of the commutator or of the product of operators is the sine (respectively, exponential) function of the bidifferential operator of the Poisson bracket applied to the corresponding symbols.

In the early 70's, Berezin was developing the general mathematical definition of quantization as a kind of a functor from the category of classical mechanics to a certain category of associative algebras [5]. In particular, he considered quantization on Kähler manifolds. However, Berezin's approach was analytic in nature and it required very restrictive geometric conditions on the underlying Kähler structure [5]. As a result, the only meaningful examples established in [5] were $\mathbb{C}^{n}$ and homogeneous bounded domains in $\mathbb{C}^{n}$. Later on, Berezin's approach and its relation with geometric quantization were developed in [6, 8]. Only recently in [8] A. Karabegov, using local operator-type constructions, has proved that on an arbitrary Kähler manifold there exists a special deformation quantization (with "separation of variables") which is local and is parameterized by an arbitrary formal deformation of the Kähler potential.

About the same time as Berezin, Bayen, Flato, Fronsdal, Lichnerowicz and Sternheimer [9, 10 considered quantization as a deformation of the usual commutative product of classical observables into a noncommutative associative product $\star_{\hbar}$ which is parameterized by the Planck constant $\hbar$ and satisfies the correspondence principle. In [9, 10 they systematically developed the concept of deformation quantization as a theory of $\star$ - products and gave an independent formulation of quantum mechanics based on this notion. The BFFLS approach was inspired by the work of Vey [11] on the deformation of Poisson structures, where the idea of the $\star$ product had already appeared. Subsequently, the work [9, 10] laid a foundation for the deformation quantization, a theory that utilizes pure algebraic approach 
based on Hochschild cohomology to the deformation of associative algebras, developed by Gerstenhaber 12], together with the Chevalley-Eilenberg cohomology of the Poisson-Lie algebra of classical observables.

Because of its physical origin and motivation, the problem of deformation quantization was first considered for symplectic manifolds. First results in this direction were theorems by Vey [11] and Neroslavsky and Vlasov 13 that deformations of the Poisson-Lie algebra and of the commutative algebra of classical observables exist whenever the third Betti number of a symplectic manifold vanishes. It was shown in [14] that for any symplectic manifold there exists a fourth order quantization. Finally, the problem of existence and classification of $\star$ - products on arbitrary symplectic manifolds was solved by De Wilde-Lecomte [15, 16]. Another approach was taken in [17, 18] and Fedosov [19. In particular, in [19] a rather explicit construction of the $\star$ - product was given.

The problem of deformation quantization is naturally formulated for the Poisson manifolds as well. Until recently, the best known example of such quantization was the Etinhof-Kazhdan theorem 20] that every Poisson-Lie group can be quantized. The situation changed when M. Kontsevich proved [21] his famous formality conjecture, which, in particular, implies that deformation quantization exists on every Poisson manifold. In addition to this general result, Kontsevich presented a universal formula for the deformation quantization of an arbitrary Poisson structure on $\mathbb{R}^{n}$. This remarkable formula gives a $\star$ - product of smooth functions on $\mathbb{R}^{n}$ as formal power series in $\hbar$ of bidifferential operators parameterized by graphs, with coefficients given by integrals over certain configuration spaces. As was stated in [21], this series resembles a perturbation expansion of the topological sigmamodel of B type (after a suitable supersymmetry transformation). Recently, this perturbation expansion was carried through in [22].

The present paper appeared from an attempt to understand further the analogy with the perturbation expansion. Specifically, we observe that there exists a simple integral formula for the deformation quantization on Kähler manifolds based on the finite-dimensional formal integral, defined through the Laplace method approximation. Quite naturally, coefficients of the corresponding formal power series can be written in terms of "Feynman graphs".

Remarkably, the integral that we consider is almost identical to the one used by Berezin [5]. The difference, however, is that now we regard it as a formal power series in the deformation parameter $\hbar$, and our approach works for an arbitrary Kähler manifold, whereas Berezin was considering a $\star$ - product given by a convergent integral in some complex domain. As the result, he was forced to impose certain global restrictions on the Kähler manifold, which make his approach meaningful only for $\mathbb{C}^{n}$ with the standard Kähler structure and for flag varieties, as discussed in [6, 7] (and in [24] for generalized flag varieties). In comparison, our approach gives a direct proof of the theorem proved by Karabegov [8] in terms of Berezin's original integral.

The organization of the paper is as follows. In section 2 we recall some basic facts from Berezin's paper [5]. In the next section we construct the non-normalized $\star$ - product: deformed product for Kähler manifolds, given by a simple (formal) integral formula, which does not preserve the unit element of the commutative algebra of classical observables. In section 4 we present the normalized $\star$ - product, which preserves the unit element, and show that there exists formal Bergman kernel. 
In the appendix, we prove the equality between two types of the formal integrals introduced in the paper.

\section{BEREZIN's QUANTIZATION}

2.1. Covariant symbols. Consider a complex manifold $\mathbb{C}^{n}$ endowed with a Kähler metric

$$
d s^{2}=\sum_{i, j=1}^{n} h_{i \bar{j}} d z^{i} \otimes d \bar{z}^{j}
$$

Since $\mathbb{C}^{n}$ is a two-connected Stein manifold, every Kähler metric on it admits a global potential $\Phi(z, \bar{z})$, i.e.

$$
h_{i \bar{j}}=\frac{\partial^{2} \Phi}{\partial z^{i} \partial \bar{z}^{j}},
$$

where we are using complex coordinates $z^{i}, \bar{z}^{i}, i=1, \ldots, n$, on $\mathbb{C}^{n}$. Let

$$
\omega=-\frac{1}{2} \operatorname{Im} d s^{2}=\frac{\sqrt{-1}}{2} \sum_{i, j=1}^{n} h_{i \bar{j}} d z^{i} \wedge d \bar{z}^{j},
$$

be the corresponding symplectic form. It induces a Poisson structure on the algebra $\mathcal{A}=C^{\infty}\left(\mathbb{C}^{n}\right)$ of classical observables

$$
\left\{f_{1}, f_{2}\right\}=\frac{2}{\sqrt{-1}} \sum_{i, j=1}^{n} h^{i \bar{j}}\left(\partial_{i} f_{1} \bar{\partial}_{j} f_{2}-\bar{\partial}_{j} f_{1} \partial_{i} f_{2}\right),
$$

where $\partial_{i}=\partial / \partial z^{i}, \bar{\partial}_{i}=\partial / \partial \bar{z}^{i}$ and $h^{i \bar{j}}$ are matrix elements of the inverse matrix $H^{-1}$ of the matrix $H=\left(h_{i \bar{j}}\right)$ of the Kähler metric.

Let $\hbar$ be a positive real number. Denote by $d \mu_{\hbar}=(\omega / \pi \hbar)^{n}$ normalized volume form on $\mathbb{C}^{n}$,

$$
d \mu_{\hbar}(z, \bar{z})=\operatorname{det} H \prod_{i=1}^{n} \frac{\left|d z^{i} \wedge d \bar{z}^{i}\right|}{2 \pi \hbar},
$$

and define $\mathcal{H}_{\hbar}$ as the Hilbert space of complex valued, measurable functions on $\mathbb{C}^{n}$ which are square summable with respect to the measure $e^{-\Phi(z, \bar{z}) / \hbar} d \mu_{\hbar}(z, \bar{z})$. The inner product of $f_{1}, f_{2} \in \mathcal{H}_{\hbar}$ is given by

$$
\left(f_{1}, f_{2}\right)=\int_{\mathbb{C}^{n}} f_{1}(z, \bar{z}) \overline{f_{2}(z, \bar{z})} e^{-\Phi(z, \bar{z}) / \hbar} d \mu_{\hbar}(z, \bar{z}),
$$

where in order to distinguish $C^{\infty}$ functions on $\mathbb{C}^{n}$ from the holomorphic ones, we denote the former by $f(z, \bar{z})$, and the latter by $f(z)$.

Let $\mathcal{F}_{\hbar} \subset \mathcal{H}_{\hbar}$ be the subspace of holomorphic functions and $P: \mathcal{H}_{\hbar} \rightarrow \mathcal{F}_{\hbar}$ be the corresponding orthogonal projection operator. Explicitly,

$$
(P f)(z)=\int_{\mathbb{C}^{n}} B_{\hbar}(z, \bar{v}) f(v, \bar{v}) e^{-\Phi(v, \bar{v}) / \hbar} d \mu_{\hbar}(v, \bar{v}),
$$

where $B_{\hbar}(z, \bar{v})$ is the so-called Bergman kernel. It is defined by the following absolutely and uniformly convergent series:

$$
B_{\hbar}(z, \bar{v})=\sum_{k=1}^{\infty} f_{k}(z) \overline{f_{k}(v)}
$$


for an arbitrary orthonormal basis $\left\{f_{k}\right\}$ in $\mathcal{F}_{\hbar}$ with respect to the scalar product (2.6).

Representation (2.7) shows that holomorphic functions $\Phi_{\bar{v}}(z)=B_{\hbar}(z, \bar{v})$, parameterized by $v \in \mathbb{C}^{n}$, form a complete system in $\mathcal{F}_{\hbar}$. For every bounded operator $F$ in $\mathcal{F}_{\hbar}$ define its covariant symbol 25] with respect to the complete system $\left\{\Phi_{\bar{v}}\right\}_{v \in \mathbb{C}^{n}}$ as the restriction to the diagonal $v=z$ of the following function

$$
f(z, \bar{v})=\frac{\left(F \Phi_{\bar{v}}, \Phi_{\bar{z}}\right)}{\left(\Phi_{\bar{v}}, \Phi_{\bar{z}}\right)}
$$

defined on $\mathbb{C}^{n} \times \mathbb{C}^{n}$. The function $f$ is holomorphic in $z$ and anti-holomorphic in $v$.

Denote by $\mathcal{A}_{\hbar}$ the linear space of covariant symbols of bounded operators in $\mathcal{F}_{\hbar}$; there is a natural inclusion $\mathcal{A}_{\hbar} \subset \mathcal{A}$. Then, according to [5], we have the following result.

Lemma 2.1. The space of covariant symbols $\mathcal{A}_{\hbar}$ is a $\mathbb{C}$-algebra (an associative algebra over $\mathbb{C}$ with unit), with the $\star$ - product given by the following integral

$$
\left(f_{1} \star_{\hbar} f_{2}\right)(z, \bar{z})=\int_{\mathbb{C}^{n}} f_{1}(z, \bar{v}) f_{2}(v, \bar{z}) \frac{B_{\hbar}(z, \bar{v}) B_{\hbar}(v, \bar{z})}{B_{\hbar}(z, \bar{z})} e^{-\Phi(v, \bar{v}) / \hbar} d \mu_{\hbar}(v, \bar{v}),
$$

and with the unit given by the symbol of the identity operator - function on $\mathbb{C}^{n}$, identically equal to 1 . Also, there exists a trace functional $\operatorname{tr}: \mathcal{A}_{\hbar}^{0} \rightarrow \mathbb{C}$, defined on symbols of bounded operators of the trace class by the formula

$$
\operatorname{tr} f=\int_{\mathbb{C}^{n}} f(z, \bar{z}) B_{\hbar}(z, \bar{z}) e^{-\Phi(z, \bar{z}) / \hbar} d \mu_{\hbar}(z, \bar{z}),
$$

and it is cyclically invariant

$$
\operatorname{tr}\left(f_{1} \star_{\hbar} f_{2}\right)=\operatorname{tr}\left(f_{2} \star_{\hbar} f_{1}\right) .
$$

Proof. It is a straightforward computation; see [5, 25] for details. The associativity of the $\star$ - product follows from the associativity of the operator product. It can be also verified directly from the integral (2.10) using the Fubini theorem; all issues regarding convergence, etc., are standard. The same applies to the cyclic trace property. Finally, the property of the unit follows from the definition of the Bergman kernel.

2.2. Contravariant symbols. A bounded operator $F$ in $\mathcal{F}_{\hbar}$ is called a BerezinToeplitz operator, if $F=P \hat{f}$, where $\hat{f}$ is a multiplication operator by a function $\hat{f} \in \mathcal{H}_{\hbar}$, called the contravariant symbol of $F$. Covariant symbol $f$ of the operator $F$ can be expressed through its contravariant symbol $\hat{f}$ (if it exists) as follows [25]

$$
f(z, \bar{z})=\int_{\mathbb{C}^{n}} \hat{f}(v, \bar{v}) \frac{B_{\hbar}(z, \bar{v}) B_{\hbar}(v, \bar{z})}{B_{\hbar}(z, \bar{z})} e^{-\Phi(v, \bar{v}) / \hbar} d \mu_{\hbar}(v, \bar{v}) .
$$

The proof is a straightforward computation. The correspondence $\hat{f} \mapsto f$ defines a linear operator $I_{\hbar}: \mathcal{F}_{\hbar} \rightarrow \mathcal{F}_{\hbar}, I_{\hbar}(\hat{f})=f$.

Lemma 2.2. If $F$ is a Berezin-Toeplitz operator in $\mathcal{F}_{\hbar}$ with contravariant symbol $\hat{f}$, then for all $g \in \mathcal{F}_{\hbar}$ we have 25]

$$
F g(z)=\int_{\mathbb{C}^{n}} B_{\hbar}(z, \bar{v}) f(z, \bar{v}) g(v) e^{-\Phi(v, \bar{v}) / \hbar} d \mu_{\hbar}(v, \bar{v}),
$$


where $f$ is the covariant symbol of $F$, given by the formula (2.12). Also, the product of Berezin-Toeplitz operators $F_{1}$ and $F_{2}$ with contravariant symbols $\hat{f}_{1}$ and $\hat{f}_{2}$ can be expressed through their covariant symbols $f_{1}$ and $f_{2}$ by the following formula:

$$
\left(F_{1} F_{2}\right) g(z)=\int_{\mathbb{C}^{n}} B_{\hbar}(z, \bar{v})\left(f_{1} \star f_{2}\right)(z, \bar{v}) e^{-\Phi(v, \bar{v}) / \hbar} d \mu_{\hbar}(v, \bar{v}) .
$$

Proof. It is another straightforward computation, based on the following expression of $\star$-product of covariant symbols through corresponding contravariant ones,

$$
\begin{aligned}
\left(f_{1} \star_{\hbar} f_{2}\right)(z, \bar{z})= & \iint_{\mathbb{C}^{n} \times \mathbb{C}^{n}} \hat{f}_{1}(v, \bar{v}) \hat{f}_{2}(w, \bar{w}) \frac{B_{\hbar}(z, \bar{v}) B_{\hbar}(v, \bar{w}) B_{\hbar}(w, \bar{z})}{B_{\hbar}(z, \bar{z})} \\
& \times e^{-(\Phi(v, \bar{v})+\Phi(w, \bar{w})) / \hbar} d \mu_{\hbar}(v, \bar{v}) d \mu_{\hbar}(w, \bar{w}) .
\end{aligned}
$$

If the linear operator $I_{\hbar}$ were one-to-one and onto, equation $(2.15)$ would allow to introduce the product $\hat{\star}_{\hbar}$ of contravariant symbols as follows

$$
\hat{f}_{1} \hat{\star}_{\hbar} \hat{f}_{2}=I_{\hbar}^{-1}\left(I_{\hbar}\left(\hat{f}_{1}\right) \star_{\hbar} I_{\hbar}\left(\hat{f}_{2}\right)\right) \text {. }
$$

In general [5, 25], however, the operator $I_{\hbar}$ is not onto, since there exist bounded operators which do not have contravariant symbols.

2.3. The correspondence principle. To consider the family of associative algebras $\left\{\mathcal{A}_{\hbar}\right\}_{\hbar>0}$ as a quantization of the Poisson algebra of classical observables $\mathcal{A}=C^{\infty}\left(\mathbb{C}^{n}\right)$, the "correspondence principle"

$$
\lim _{\hbar \rightarrow 0} \frac{2 \sqrt{-1}}{\hbar}\left(f_{1} \star_{\hbar} f_{2}-f_{2} \star_{\hbar} f_{1}\right)=\left\{f_{1}, f_{2}\right\}
$$

should be valid for "suitable" continuous families $f_{i}(z, \bar{z} ; \hbar) \in \mathcal{A}_{\hbar} \subset \mathcal{A}$, where in the right-hand side $f_{i}(z, \bar{z})=\left.f_{i}(z, \bar{z} ; \hbar)\right|_{\hbar=0} \in \mathcal{A}, i=1,2$.

In order to verify (2.17) and to introduce these families of symbols, Berezin [5] made several assumptions.

First, he assumed that Kähler potential $\Phi(z, \bar{z})$ admits an analytic continuation $\Phi(z, \bar{v})$ to $\mathbb{C}^{n} \times \mathbb{C}^{n}$. Setting

$$
B_{\hbar}(z, \bar{v})=e^{\Phi(z, \bar{v}) / \hbar} e_{\hbar}(z, \bar{v}),
$$

one can rewrite the integral (2.10) as follows

$$
\left(f_{1} \star_{\hbar} f_{2}\right)(z, \bar{z})=\int_{\mathbb{C}^{n}} f_{1}(z, \bar{v}) f_{2}(v, \bar{z}) \frac{e_{\hbar}(z, \bar{v}) e_{\hbar}(v, \bar{z})}{e_{\hbar}(z, \bar{z})} e^{\phi(z, \bar{z} ; v, \bar{v}) / \hbar} d \mu_{\hbar}(v, \bar{v}),
$$

where $\phi(z, \bar{z} ; v, \bar{v})=\Phi(z, \bar{v})+\Phi(v, \bar{z})-\Phi(z, \bar{z})-\Phi(v, \bar{v})$ is the so-called Calabi diastatic function of the Kähler form $\omega$.

Remark 2.3. When Kähler form $\omega$ is real-analytic, the Calabi function is globally defined in some neighborhood of the diagonal in $\mathbb{C}^{n} \times \mathbb{C}^{n}$. In general, for an arbitrary Kähler manifold with a real-analytic Kähler form, the Calabi function is defined in some neighborhood of the diagonal in $M \times M$.

The exponential factor in integral (2.19) has a critical point at $v=z$, so that the Laplace method is applicable (for smooth functions $f_{1}$ and $f_{2}$ ), provided that the asymptotic behavior of $e_{\hbar}$ as $\hbar \rightarrow 0$ is also known. In [5], Berezin did not consider this problem, which we address and solve in section 4 . Instead, Berezin made the second assumption (hypothesis A-D in [5]), that $e_{\hbar}(z, \bar{v})=1$ for all $z, v \in \mathbb{C}^{n}$. 
This condition is very restrictive. It implies (see [5], Theorem 2.5) that log det $H$ is harmonic, so that Berezin's quantization works only for very special Kähler manifolds, essentially, for the flag varieties and their generalizations. The corresponding construction depends on a particular choice of the Kähler potential and is coordinate dependent.

\section{NON-NORMALIZED $\star$ - PRODUCT FOR KÄHLER MANIFOLDS}

3.1. Non-normalized $\star$ - product for $\mathbb{C}^{n}$. Let $\Phi(z, \bar{v})$ be analytical continuation of $\Phi(z, \bar{z})$ to $\mathbb{C}^{n} \times \mathbb{C}^{n}$ and let $\phi(z, \bar{z} ; v, \bar{v})=\Phi(z, \bar{v})+\Phi(v, \bar{z})-\Phi(z, \bar{z})-\Phi(v, \bar{v})$ be its Calabi function. The following statement holds.

Lemma 3.1. The point $v=z$ is a critical point for the Calabi function $\phi(z, \bar{z} ; v, \bar{v})$ considered as a function of $v$ and $\bar{v}$. One has the following expansion

$$
\phi(z, \bar{z} ; v, \bar{v})=-\sum_{|I|+|J|>1} \frac{1}{I ! J !} \Phi_{I \bar{J}}(v-z)^{I}(\bar{v}-\bar{z})^{J}
$$

with the standard notation for the multi-indices $I=\left(i_{1}, \ldots, i_{n}\right), J=\left(j_{1}, \ldots, j_{n}\right)$,

$$
\begin{aligned}
|I| & =i_{1}+\cdots+i_{n},|J|=j_{1}+\cdots+j_{n}, I !=i_{1} ! \cdots i_{n} !, J !=j_{1} ! \cdots j_{n} !, \\
(v-z)^{I} & =\left(v^{1}-z^{1}\right)^{i_{1}} \cdots\left(v^{n}-z^{n}\right)^{i_{n}},(\bar{v}-\bar{z})^{J}=\left(\bar{v}^{1}-\bar{z}^{1}\right)^{j_{1}} \cdots\left(\bar{v}^{n}-\bar{z}^{n}\right)^{j_{n}} .
\end{aligned}
$$

Here

$$
\Phi_{I \bar{J}}=\partial^{I} \bar{\partial}^{J} \Phi=\partial^{I^{\prime}} \bar{\partial}^{J^{\prime}} h_{i_{1} \overline{j_{1}}},
$$

where $I^{\prime}=I \backslash\left\{i_{1}\right\}, J^{\prime}=J \backslash\left\{j_{1}\right\}$ and $\partial^{I}=\partial_{1}^{i_{1}} \cdots \partial_{n}^{i_{n}}, \bar{\partial}^{J}=\bar{\partial}_{1}^{j_{1}} \cdots \bar{\partial}_{n}^{j_{n}}$. In particular, $\Phi_{i \bar{j}}=\partial_{i} \bar{\partial}_{j} \Phi=h_{i \bar{j}}$.

This lemma shows that coefficients of the Taylor expansion of $\phi$ at the critical point $v=z$ depend only on the Kähler metric. The following definition is thus justified.

Definition 3.2. The non-normalized $\star$ - product is a $\mathbb{C}[[h]]$-linear map

$$
\bullet: \mathcal{A}[[\hbar]] \otimes \mathcal{A}[[\hbar]] \rightarrow \mathcal{A}[[\hbar]],
$$

defined by the following formal integral

$$
\left(f_{1} \bullet f_{2}\right)(z, \bar{z})=\int_{\mathbb{C}^{n}} f_{1}(z, \bar{v}) f_{2}(v, \bar{z}) e^{\phi(z, \bar{z} ; v, \bar{v}) / \hbar} d \mu_{\hbar}(v, \bar{v}),
$$

i.e. the formal power series in $\hbar$ that arises from the Laplace expansion at the critical point $v=z$.

Remark 3.3. In the formal integral (3.1) there is no need to assume that functions $f_{1}$ and $f_{2}$ admit analytic continuation into $\mathbb{C}^{n} \times \mathbb{C}^{n}$. The definition of the $\bullet$ - product uses the formal expansion around the diagonal in $\mathbb{C}^{n} \times \mathbb{C}^{n}$ and depends only on the values of $f_{1}$ and $f_{2}$ and of their partial derivatives on the diagonal. Specifically, the integral can be written as the following formal power series in $\epsilon^{2}=\hbar$,

$$
\left(f_{1} \bullet f_{2}\right)(z, \bar{z})=\int_{\mathbb{C}^{n}} f_{1}(z, \bar{z}+\epsilon \bar{y}) f_{2}(z+\epsilon y, \bar{z}) e^{\phi(z, \bar{z} ; z+\epsilon y, \bar{z}+\epsilon \bar{y}) / \epsilon^{2}} d \mu(y, \bar{y}) .
$$

where $\phi(z, \bar{z} ; z+\epsilon y, \bar{z}+\epsilon \bar{y})$ is the formal power series in $\epsilon$ determined by lemma 3.1 , $f_{1}(z, \bar{z}+\epsilon \bar{y})$ and $f_{2}(z+\epsilon y, \bar{z})$, are given by their formal power series expansions, 
$d \mu(y, \bar{y})=(\omega / \pi)^{n}$ and Gaussian integrations have been performed (see (3.4) in the next subsection). Equivalently, the formal integral (3.1) can be defined by the asymptotic of the distribution $\exp (\phi(z, \bar{z} ; v, \bar{v}) / \hbar)$ as $\hbar \rightarrow 0$.

Lemma 3.4. The product $\bullet$ is associative,

$$
\left(f_{1} \bullet f_{2}\right) \bullet f_{3}=f_{1} \bullet\left(f_{2} \bullet f_{3}\right),
$$

for all $f_{1}, f_{2}, f_{3} \in \mathcal{A}$.

Proof. It follows from the definition of the Calabi function that

$$
\phi(z, \bar{w}, v, \bar{v})+\phi(z, \bar{z}, w, \bar{w})=\phi(v, \bar{z}, w, \bar{w})+\phi(z, \bar{z}, v, \bar{v}) .
$$

Therefore, both sides of the associativity equation are equal to the same formal integral

$$
\iint_{\mathbb{C}^{n} \times \mathbb{C}^{n}} f_{1}(z, \bar{v}) f_{2}(v, \bar{w}) f_{3}(w, \bar{z}) e^{(\phi(z, \bar{z} ; v, \bar{v})+\phi(v, \bar{z} ; w, \bar{w})) / \hbar} d \mu_{\hbar}(v, \bar{v}) d \mu_{\hbar}(w, \bar{w}),
$$

provided that one has the formal analog of the Fubini theorem: "double formal integral equals to the repeated formal integral".

The latter statement can be proved as follows. First, it is clearly valid for convergent integrals, provided that all sufficient conditions for the uniform convergence are satisfied. These conditions are in the form of inequalities for the coefficients $\Phi_{I \bar{J}}$ and for the Taylor coefficients of $f_{1}, f_{2}, f_{3}$. Since the associativity equation can be written as a system of polynomial equations for these coefficients, these equations hold for arbitrary values of the coefficients involved - "the nonessential property of algebraic identities" principle.

3.2. Feynman graphs and coefficients of non-normalized $\star$ - product for $\mathbb{C}^{n}$. Here we represent the formal power series for the $\bullet$ - product using Feynman graphs (see any textbook on quantum field theory; for a mathematical exposition see, e.g. [23).

Let $\Gamma$ be a graph, i.e. a finite one-dimensional simplicial complex with oriented edges. The graph $\Gamma$ is locally oriented if an enumeration of identically oriented edges is fixed around each vertex.

Consider a locally oriented graph $\Gamma$ equipped with the following data.

(i) For each edge $e \in \Gamma_{1}$ of $\Gamma$ there are given a finite-dimensional vector space $V_{e}$ over $\mathbb{C}$, its dual space $V_{e}^{\prime}$, and a linear map $L_{e}: V_{e}^{\prime} \rightarrow V_{e}$.

(ii) For each vertex $v \in \Gamma_{0}$ of $\Gamma$ there is given a linear map

$$
\gamma_{v}: V_{e_{1 v}^{-}} \otimes \cdots \otimes V_{e_{n v}^{-}} \rightarrow V_{e_{1 v}^{+}}^{\prime} \otimes \cdots \otimes V_{e_{m v}^{+}}^{\prime}
$$

equivariant with respect to the symmetric group, which acts by permuting the factors in the tensor product. Here $e_{i v}^{-}, i=1, \ldots, n$, are incoming edges, and $e_{j v}^{+}, j=1, \ldots, m$, are outgoing edges in the star $\Gamma(v)$ of the vertex $v$. Also, it is assumed that the tensor product of vector spaces parameterized by the empty set is $\mathbb{C}$.

To define the partition function of a locally oriented graph $\Gamma$ with data, choose for every edge $e \in \Gamma_{1}$ a basis $\left\{\varepsilon_{i_{e}}\right\}_{i_{e}=1}^{\operatorname{dim} V_{e}}$ in the vector space $V_{e}$ and let $\left\{\varepsilon^{i_{e}}\right\}_{i_{e}=1}^{\operatorname{dim} V_{e}}$ be the dual basis in $V_{e}^{\prime}$. Denote by $\left(\gamma_{v}\right)_{I_{-} I_{+}},\left(L_{e}\right)^{k_{e} l_{e}}$, where $I_{-}=\left\{i_{e_{1 v}^{-}}, \ldots, i_{e_{n v}^{-}}\right\}$and 
$I_{+}=\left\{i_{e_{1 v}^{+}}, \ldots, i_{e_{m v}^{+}}\right\}$, the corresponding matrix elements of the linear maps $\gamma_{v}$ and $L_{e}$, and set $N=h_{1}(\Gamma) \stackrel{\text { def }}{=} \#\left(\Gamma_{1}\right)$. Then the partition function of $\Gamma$ is given by the following expression,

$$
W_{\Gamma}=W_{\Gamma}\left(\left\{\gamma_{v}\right\},\left\{V_{e}\right\},\left\{L_{e}\right\}\right) \stackrel{\operatorname{def}}{=} \sum_{i_{e_{1}}=1}^{\operatorname{dim} V_{e_{1}}} \cdots \sum_{i_{e_{N}}=1}^{\operatorname{dim} V_{e_{N}}} \prod_{v \in \Gamma_{0}}\left(\gamma_{v}\right)_{I_{-} I_{+}} \prod_{e \in \Gamma_{1}}\left(L_{e}\right)^{k_{e} l_{e}},
$$

where $k_{e}=i_{e_{p v}^{+}}$for the unique $v \in \Gamma_{0}$ such that $e=e_{p v}^{+}$and $l_{e}=i_{e_{q v}^{-}}$for the unique $v \in \Gamma_{0}$ such that $e=e_{q v}^{-}$.

Due to the equivariance property of maps $\gamma_{v}$, the partition function $W_{\Gamma}$ does not depend on the ordering of identically oriented edges around each vertex of the graph $\Gamma$. It also does not depend on the choice of bases in vector spaces $V_{e}$, and is an invariant of the locally oriented graph $\Gamma$ with data.

The partition functions of graphs with data are building blocks for expressing the asymptotics of integrals through Feynman diagrams. Specifically, consider the formal integral (3.1) which, by remark 3.3, we rewrite as follows,

$$
\pi^{-n} \int_{\mathbb{C}^{n}} f_{1}(z, \bar{z}+\varepsilon \bar{y}) f_{2}(z+\varepsilon y, \bar{z}) e^{-(H y, y)+V(z, \bar{z} ; y, \bar{y} ; \hbar)} \prod_{i=1}^{n} \frac{\left|d y^{i} \wedge d \bar{y}^{i}\right|}{2} .
$$

Here $\varepsilon=\sqrt{\hbar}$ and

$$
(H y, y)=\sum_{i, j=1}^{n} h_{i \bar{j}}(z, \bar{z}) y^{i} \bar{y}^{j}
$$

where $H=\left(h_{i \bar{j}}\right)$ is the matrix of the Kähler metric, and

$$
\begin{aligned}
V(z, \bar{z} ; y, \bar{y} ; \hbar)= & -\sum_{|I|+|J|>2} \frac{\hbar^{(|I|+|J|-2) / 2}}{I ! J !} \Phi_{I \bar{J}}(z, \bar{z}) y^{I} \bar{y}^{J} \\
& +\sum_{|I|,|J| \geq 0} \frac{\hbar^{(|I|+|J|) / 2}}{I ! J !} \Psi_{I \bar{J}}(z, \bar{z}) y^{I} \bar{y}^{J}
\end{aligned}
$$

where we are using notations of Lemma 3.1 and

$$
\Psi_{I \bar{J}}(z, \bar{z})=\partial^{I} \bar{\partial}^{J} \log \operatorname{det} H(z, \bar{z})
$$

The evaluation of the formal integral (3.1) is based on the fundamental formula of Gaussian integration

$$
\int_{\mathbb{C}^{n}} y^{I} \bar{y}^{J} e^{-(H y, y)} \prod_{i=1}^{n} \frac{\left|d y^{i} \wedge d \bar{y}^{i}\right|}{2}=\left.\frac{\pi^{n}}{\operatorname{det} H}\left(\partial^{I} \bar{\partial}^{J} e^{\left(H^{-1} z, z\right)}\right)\right|_{z=\bar{z}=0} .
$$

Namely, expand $f_{1}(z, \bar{z}+\varepsilon \bar{y}) f_{2}(z+\varepsilon y, \bar{z}) \exp V(z, \bar{z} ; y, \bar{y} ; \hbar)$ in power series in $\varepsilon$ and evaluate each term using (3.4). As a result, one gets an expression of (3.1) as a formal power series in $\hbar=\varepsilon^{2}$ with coefficients given as sums over a subset $\mathcal{G}$ of the set of all locally oriented graphs.

This subset $\mathcal{G}$ is characterized by the two conditions. First, for every $\Gamma \in \mathcal{G}$, the set $\Gamma_{0}$ always contains two special elements: a vertex $R$ with only incoming edges, and a vertex $L$ with only outgoing edges (see Fig. 1).

Second, the complement of the set $\{L, R\}$ in $\Gamma_{0}$ consists of vertices of two types, "solid" and "hollow", either one or both of these sets may be empty (see Fig. 2). 

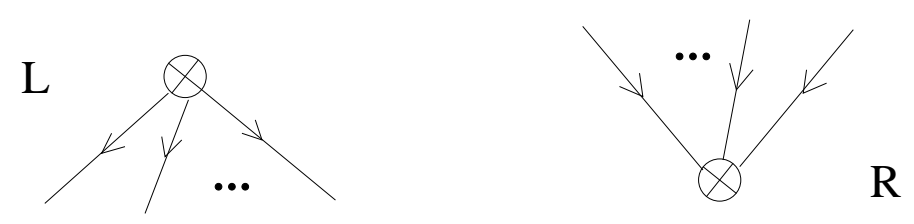

FIGURE 1.
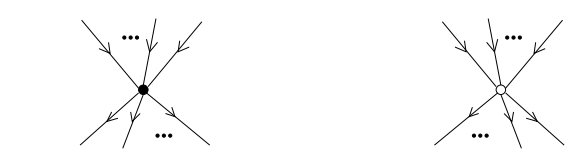

"Solid" vertices

"Hollow" vertices

FIGURE 2.

For every $\Gamma \in \mathcal{G}$ let $\operatorname{Aut}(\Gamma)$ be the subgroup of the group of automorphisms of $\Gamma$ that maps vertices to the vertices of the same type and preserves $L$ and $R$ vertices and let $|\operatorname{Aut}(\Gamma)|=\# \operatorname{Aut}(\Gamma)$. Also set $\chi(\Gamma)=\#\left(\Gamma_{1}\right)-\#\left(\Gamma_{0}^{s}\right)$, where $\Gamma_{0}^{s} \subset \Gamma_{0}$ is the subset of "solid" vertices.

The next theorem summarizes the description of the formal integral (3.1) in terms of Feynman diagrams.

Theorem 3.5. The non-normalized $\star$ - product $\bullet$ is given by

$$
f_{1} \bullet f_{2}=\sum_{\Gamma \in \mathcal{G}} \frac{\hbar^{\chi(\Gamma)}}{|\operatorname{Aut}(\Gamma)|} D_{\Gamma}\left(f_{1}, f_{2}\right)
$$

where $D_{\Gamma}\left(f_{1}, f_{2}\right)=W_{\Gamma}$ is the partition function of the graph $\Gamma \in \mathcal{G}$ with the following data.

1. For each edge $e \in \Gamma_{1}$ there are given a vector space $V=\mathbb{C}^{n}$ with the standard basis $\left\{\varepsilon_{i}\right\}$, its dual space $V^{\prime}=\mathbb{C}^{n}$ with the dual basis $\left\{\varepsilon^{\bar{i}}\right\}$ and a linear map $L: V^{\prime} \rightarrow V$, defined by $K\left(\varepsilon^{\bar{j}}\right)=\sum_{i=1}^{n} h^{i \bar{j}}(z, \bar{z}) \varepsilon_{i}$, where $h^{i \bar{j}}$ are elements of the matrix $H^{-1}$.

2. For each "solid" vertex $v_{s}$, with $n$ incoming and $m$ outgoing edges, there is given a linear map $\gamma_{v_{s}}: V^{\otimes n} \rightarrow\left(V^{\prime}\right)^{\otimes m}$, defined by the matrix with elements $-\Phi_{I \bar{J}}(z, \bar{z})$.

3. For each "hollow" vertex $v_{h}$, with $n$ incoming and $m$ outgoing edges, there is given a linear map $\gamma_{v_{h}}: V^{\otimes n} \rightarrow\left(V^{\prime}\right)^{\otimes m}$, defined by the matrix with elements $\Psi_{I \bar{J}}(z, \bar{z})$.

4. For the special vertex $R$, with $n$ incoming edges, there is given a linear map $\gamma_{R}: V^{\otimes n} \rightarrow \mathbb{C}$, defined by the matrix with elements $f_{2 I}(z \bar{z})=\partial^{I} f_{2}(z, \bar{z})$.

5 . For the special vertex $L$, with $m$ outgoing edges, there is given a linear map $\gamma_{L}: \mathbb{C} \rightarrow\left(V^{\prime}\right)^{\otimes n}$ defined by the matrix with elements $f_{1 \bar{J}}(z, \bar{z})=\bar{\partial}^{J} f_{1}(z, \bar{z})$.

Remark 3.6. Elements of linear maps assigned to solid and hollow vertices are both expressed through the Kähler metric and, therefore, are related. These relations are obtained from the fundamental one

$$
\partial_{i} \log \operatorname{det} H=\operatorname{tr}\left(H^{-1} \partial_{i} H\right),
$$

which is represented graphically in Figure 3, by successive differentiation. 


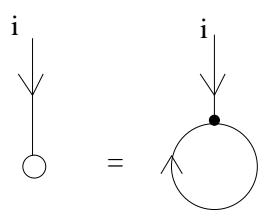

Figure 3.

Typical examples are

$$
\partial_{i j}^{2} \log \operatorname{det} H=\operatorname{tr}\left(H^{-1} \partial_{i j}^{2} H\right)-\operatorname{tr}\left(H^{-1} \partial_{i} H H^{-1} \partial_{j} H\right),
$$

depicted as equality of weights of graphs in Figure 4, and

$$
\begin{aligned}
\partial_{i j k}^{3} \log \operatorname{det} H & =\operatorname{tr}\left(H^{-1} \partial_{i} H H^{-1} \partial_{j} H H^{-1} \partial_{k} H\right) \\
& -\operatorname{tr}\left(H^{-1} \partial_{i j}^{2} H H^{-1} \partial_{k} H\right)+\operatorname{tr}\left(H^{-1} \partial_{j} H H^{-1} \partial_{k} H H^{-1} \partial_{i} H\right) \\
& -\operatorname{tr}\left(H^{-1} \partial_{i k}^{2} H H^{-1} \partial_{j} H\right)-\operatorname{tr}\left(H^{-1} \partial_{i} H H^{-1} \partial_{j k}^{2} H\right) \\
& +\operatorname{tr}\left(H^{-1} \partial_{i j k}^{3} H\right),
\end{aligned}
$$

presented in Figure 5.

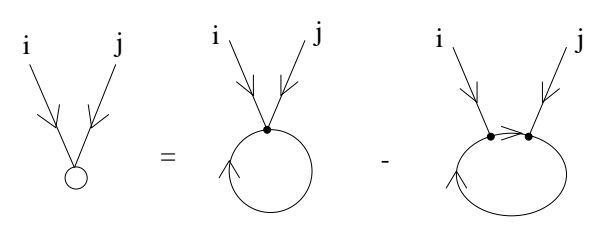

Figure 4.

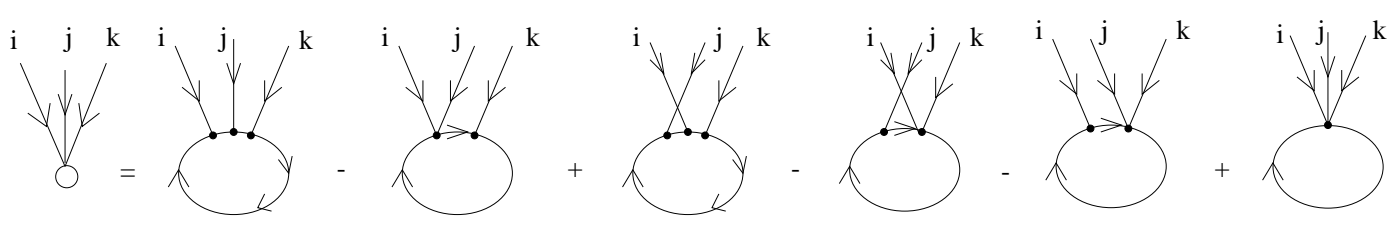

FiguRE 5. 
Lemma 3.7. The first two terms of the product $f_{1} \bullet f_{2}$ are given by

$$
\begin{aligned}
f_{1} \bullet f_{2} & =f_{1} f_{2}+\hbar\left(A f_{1} f_{2}+\sum_{i, j=1}^{n} h^{i \bar{j}} \bar{\partial}_{j} f_{1} \partial_{i} f_{2}\right) \\
& +\frac{\hbar^{2}}{2}\left(\sum _ { i , j = 1 } ^ { n } \sum _ { k , l = 1 } ^ { n } \left(h^{i \bar{j}} h^{k \bar{l}} \bar{\partial}_{j l}^{2} f_{1} \partial_{i k}^{2} f_{2}\right.\right. \\
& \left.+h^{i \bar{j}} \partial_{i} h^{k \bar{l}} \bar{\partial}_{j l}^{2} f_{1} \partial_{k} f_{2}+h^{i \bar{j}} \bar{\partial}_{j} h^{k \bar{l}} \bar{\partial}_{l} f_{1} \partial_{i k}^{2} f_{2}\right) \\
& +\sum_{i, j=1}^{n} h^{i \bar{j}}\left(\bar{\partial}_{j}\left(A f_{1}\right) \partial_{i} f_{2}+\bar{\partial}_{j} f_{1} \partial_{i}\left(A f_{2}\right)\right. \\
& \left.+\sum_{i, j=1}^{n} A h^{i \bar{j}} \bar{\partial}_{j} f_{1} \partial_{i} f_{2}+2 D f_{1} f_{2}\right)+O\left(\hbar^{3}\right) .
\end{aligned}
$$

Here

$$
A=\frac{1}{2} \sum_{i, j=1}^{n} h^{i \bar{j}} \partial_{i} \bar{\partial}_{j} \log \operatorname{det} H,
$$

and the term $\hbar^{2} D f_{1} f_{2}$ is the contribution from the vacuum diagrams, i.e., graphs with no edges adjacent to special vertices $L$ and $R$.

Proof. It is an easy computation, based on theorem 3.5 and remark 3.6 .

Diagrammatically, this expression is given in Figure 6.

Corollary 3.8. The product $\bullet$ gives a deformation quantization of the symplectic manifold $\left(\mathbb{C}^{n}, \omega\right)$ with the property $\overline{f_{1} \bullet f_{2}}=\bar{f}_{2} \bullet \bar{f}_{1}$, where $\bar{f}(z, \bar{z})=\overline{f(\bar{z}, z)}$.

Proof. As it follows from the previous lemma,

$$
\begin{aligned}
f_{1} \bullet f_{2} & =f_{1} f_{2}+O(\hbar), \\
f_{1} \bullet f_{2}-f_{2} \bullet f_{1} & =\frac{\hbar}{2 \sqrt{-1}}\left\{f_{1}, f_{2}\right\}+O\left(\hbar^{2}\right),
\end{aligned}
$$

so that the correspondence principle is valid. The symmetry with respect to the complex conjugation follows from the definition of the $\bullet$ - product.

Remark 3.9. Another way to represent the formal integral (3.1) in terms of Feynman diagrams is to rewrite it as a formal integral over the supermanifold $\Pi T \mathbb{C}^{n}$ - the total space of the tangent bundle over $\mathbb{C}^{n}$ with reversed parity of the fibers. Namely, introducing Gaussian integration over the Grassmann variables $\xi^{i}, \bar{\xi}^{i}, i=1, \cdots, n$, we get

$$
\begin{aligned}
& \int_{\mathbb{C}^{n}} f_{1}(z, \bar{v}) f_{2}(v, \bar{z}) e^{\phi(z, \bar{z} ; v, \bar{v}) / \hbar} \omega^{n} \\
= & \int_{\Pi T \mathbb{C}^{n}} f_{1}(v, \bar{z}) f_{2}(v, \bar{z}) e^{\{\phi(z, \bar{z} ; v, \bar{v}) / \hbar+(H(v, \bar{v}) \xi, \xi)\}} \prod_{i=1}^{n} \frac{\left|d z^{i} \wedge d \bar{z}^{i}\right|}{2} d \xi^{i} d \bar{\xi}^{i},
\end{aligned}
$$

where $(H \xi, \xi)=\sum_{i, j=1}^{n} h_{i \bar{j}} \xi^{i} \bar{\xi}^{j}$. Expanding into power series in $\varepsilon=\sqrt{\hbar}$ through $v=z+\varepsilon y, \bar{v}=\bar{z}+\varepsilon \bar{y}$, and using the fundamental formula of Gaussian integration, we obtain a representation of $f_{1} \bullet f_{2}$ in terms of Feynman diagrams with "bosonic" and "fermionic" vertices. 


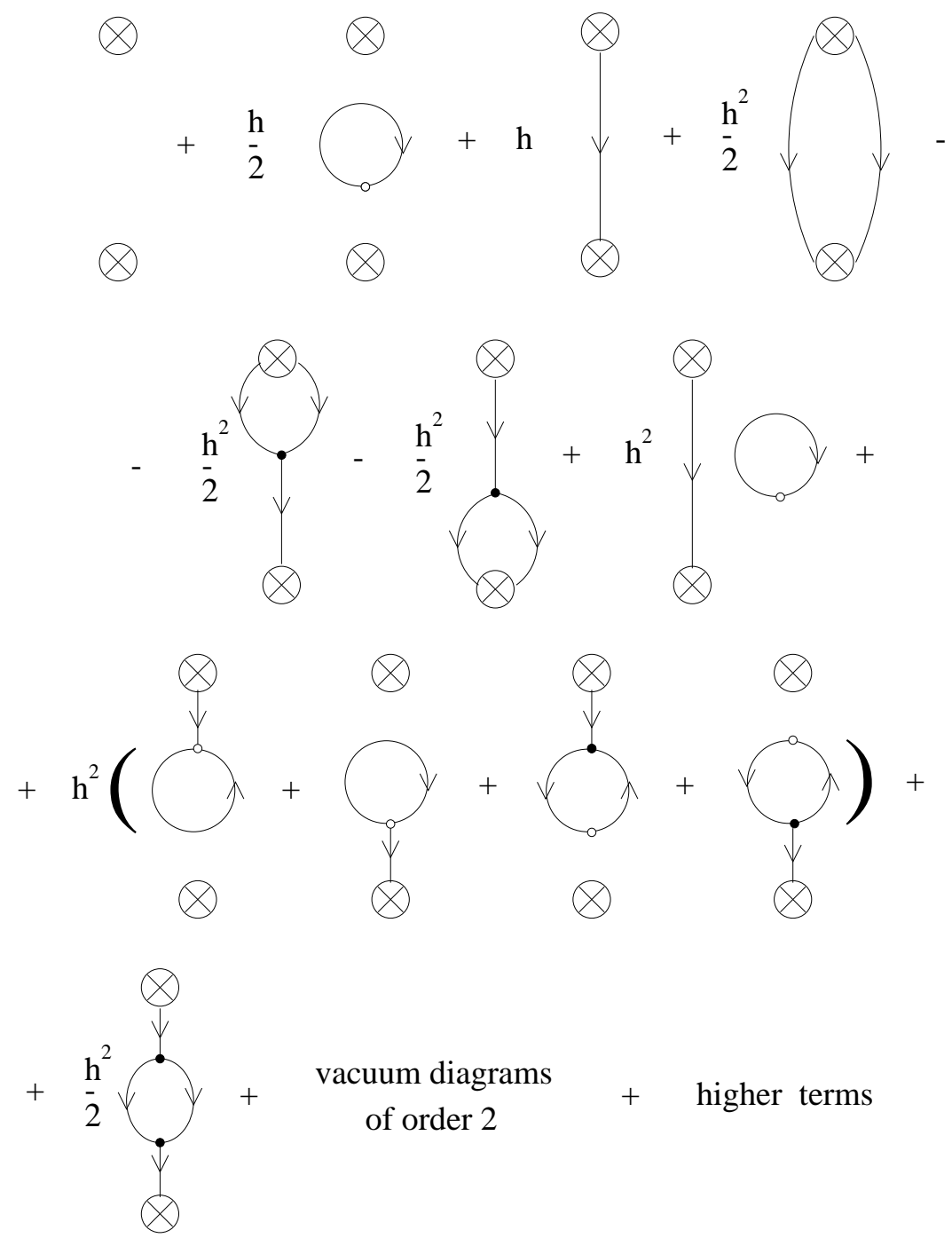

FiguRE 6.

3.3. The trace. On the subspace $\mathcal{A}_{c} \subset \mathcal{A}$ of compactly supported functions consider the following $\mathbb{C}[[\hbar]]$ - linear functional $\left.\tau: \mathcal{A}_{c}[[\hbar]] \rightarrow \mathbb{C}\left[\hbar^{-1}, \hbar\right]\right]$

$$
\tau(f)=\int_{\mathbb{C}^{n}} f(z, \bar{z}) d \mu_{\hbar}(z, \bar{z}) .
$$

Proposition 3.10. The functional $\tau$ is cyclically invariant:

$$
\tau\left(f_{1} \bullet f_{2}\right)=\tau\left(f_{2} \bullet f_{1}\right) .
$$

Proof. It follows from the definitions that $\left.\tau(f \bullet g) \in \mathbb{C}\left[\hbar^{-1}, \hbar\right]\right]$ is given by the Laplace expansion around $v=z$ of the following formal double integral:

$$
\iint_{\mathbb{C}^{n} \times \mathbb{C}^{n}} f_{1}(z, \bar{v}) f_{2}(v, \bar{z}) e^{\phi(z, \bar{z} ; v, \bar{v}) / \hbar} d \mu_{\hbar}(v, \bar{v}) d \mu_{\hbar}(z, \bar{z}),
$$


which is symmetric with respect to $v$ and $z$.

Remark 3.11. In terminology of [27], the existence of the cyclic trace functional implies that the $\bullet$ is a strongly closed $\star$ - product.

Remark 3.12. Instead of the subspace $\mathcal{A}_{c}$ of compactly supported functions on $\mathbb{C}^{n}$, one could consider a larger subspace for which the functional $\tau$ makes sense. However, this subspace would depend on the global properties of the Kähler potential $\Phi$. The subspace $\mathcal{A}_{c}$ is the maximal in $\mathcal{A}$ with the property that $\tau$ is well-defined for an arbitrary Kähler potential.

3.4. The non-normalized *-product for Kähler manifolds. Potentials for the Kähler metric $d s^{2}$ on $\mathbb{C}^{n}$ form a family $\Phi(z, \bar{z})+f(z)+\overline{f(z)}$, parameterized by entire functions $f$. However, the Calabi function $\phi(z, \bar{z} ; v, \bar{v})$ does not depend on the choice of $f$ and is a well-defined function in a neighborhood of the diagonal in $\mathbb{C}^{n} \times \mathbb{C}^{n}$ (cf. remark 2.3).

The following theorem states that the $\bullet$ - product is "functorial" with respect to the holomorphic change of coordinates.

Theorem 3.13. Let $w=g(z)$ be a holomorphic change of coordinates in a neighborhood $U \subset \mathbb{C}^{n}$ of a point $z_{0} \in \mathbb{C}^{n}$. Then

$$
\left.\left(f_{1} \bullet f_{2}\right) \circ g\right|_{U}=\left.\left(\left(f_{1} \circ g\right) \bullet\left(f_{2} \circ g\right)\right)\right|_{U},
$$

where $(f \circ g)(z, \bar{z})=f(g(z), \overline{g(z)})$.

Proof. The integral (3.1) is invariant with respect to a local change of coordinates $z \mapsto w=g(z)$ since $\phi(z, \bar{z} ; v, \bar{v})$ is a well-defined function on $U \times U$. The same applies to the power series expansion of (3.1), which is "the only thing that actually exists".

Corollary 3.14. Formula (3.1) provides a deformation quantization of an arbitrary complex symplectic manifold - a complex manifold $M$ with a symplectic form $\omega \in \Omega^{(1,1)}(M)$.

Proof. It is an immediate consequence of theorem 3.5. Consider an open covering of $M$,

$$
M=\cup_{\alpha \in A} U_{\alpha},
$$

with the property that for every $\alpha \in A$ the forms $\omega_{\alpha}=\left.\omega\right|_{U_{\alpha}}$ admit a representation (2.2)- (2.3) with some Kähler potential $\Phi_{\alpha}$. On every $U_{\alpha}$ define the $\star$ - product $f_{1} \bullet f_{2}$ of $f_{1}, f_{2} \in C^{\infty}\left(U_{\alpha}\right)$ as before. Though Kähler potentials $\Phi_{\alpha}$ on $U_{\alpha}$ do not necessarily agree on the intersections $U_{\alpha} \cap U_{\beta}$, the corresponding Calabi functions $\phi_{\alpha}$ on $U_{\alpha}$, which exist for real-analytic $\omega$, combine to a well-defined function in some neighborhood of the diagonal in $M \times M$. Applying the previous theorem in the real-analytic case, we conclude that $f_{1} \bullet f_{2} \in C^{\infty}(M)[[\hbar]]$. Since the coefficients of the $\star$ - product are polynomials in elements of $H$ and $H^{-1}$ and their partial derivatives, the same conclusion holds for the smooth case. 


\section{NORMALIZED * - PRODUCT FOR KÄHLER MANIFOLDS}

4.1. Normalized $\star$ - product for $\mathbb{C}^{n}$. Although the product $\bullet$ solves the problem of deformation quantization for complex symplectic manifolds, it has two drawbacks. First, its power series expansion is given by bidifferential operators whose coefficients contain elements of the matrix $H^{-1}$ as well as elements of $H$. This implies that the product $\bullet$ is not suitable for the deformation quantization of complex Poisson manifolds, where the coefficients should depend on the elements of the inverse matrix $H^{-1}$ only. Second, the product $\bullet$ does not preserve the unit element 1 in the commutative algebra $\mathcal{A}$.

The second problem can be easily resolved. It is well-known [26] in the deformation theory of the associative unital algebras that the unit element for the deformed product always exists. It easily follows from the fact that Hochschild cohomology classes $H^{*}(A, A)$ for a commutative $\mathbb{C}$-algebra $A$ can be represented by normalized cocycles. Indeed, normalizing the coefficients of the $\star$ - product $\bullet$ in $A[[\hbar]]$, one gets the unit element for $\bullet$ as a formal power series expressed through these coefficients. Thus in our case, the unit element is given by

$$
e_{\hbar}(z, \bar{z})=1+\sum_{l=1}^{\infty} \hbar^{l} e^{(l)}(z, \bar{z}) \in \mathcal{A}[[\hbar]],
$$

and is uniquely characterized by the equations

$$
e_{\hbar} \bullet f=f \bullet e_{\hbar}=f
$$

for all $f \in \mathcal{A}$. Because of the symmetry property with respect to the complex conjugation, only one of these equations is independent. Specifically, for the coefficients $e_{l}$ one has the following system of equations

$$
\sum_{l=0}^{\infty} \hbar^{l} \int_{\mathbb{C}^{n}} e^{(l)}(z, \bar{v}) f(v, \bar{z}) e^{\phi(z, \bar{z} ; v, \bar{v}) / \hbar} d \mu_{\hbar}(v, \bar{v})=f(z, \bar{z}),
$$

for all $f \in \mathcal{A}$.

Remark 4.1. It is not difficult to find the first few coefficients of the unit $e_{\hbar}$. Indeed, using lemma 3.7 and the notations there, we get

$$
e_{\hbar}=1-\hbar A+\hbar^{2}\left(A^{2}-D\right)+O\left(\hbar^{3}\right),
$$

so that $e_{\hbar}(z, \bar{z})$ is an invertible element in $\mathcal{A}[[\hbar]]$.

Now we can introduce the normalized $\star$ - product by twisting the non-normalized product $\bullet$ by the unit element $e_{\hbar}$.

Definition 4.2. The normalized product $\star$ is given by

$$
\left(f_{1} \star f_{2}\right)(z, \bar{z})=e_{\hbar}^{-1}(z, \bar{z})\left(\left(f_{1} e_{\hbar}\right) \bullet\left(f_{2} e_{\hbar}\right)\right)(z, \bar{z}),
$$

where $f_{1} e_{\hbar}$ and $f_{2} e_{\hbar}$ stand for the point-wise product in $\mathcal{A}[[\hbar]]$ and $e_{\hbar}^{-1} \in \mathcal{A}[[\hbar]]$.

Explicitly, the $\star$-product is given by the following formal integral

$$
\left(f_{1} \star f_{2}\right)(z, \bar{z})=\int_{\mathbb{C}^{n}} f_{1}(z, \bar{v}) f_{2}(v, \bar{z}) \frac{e_{\hbar}(z, \bar{v}) e_{\hbar}(v, \bar{z})}{e_{\hbar}(z, \bar{z})} e^{\phi(z, \bar{z} ; v, \bar{v}) / \hbar} d \mu_{\hbar}(v, \bar{v}) .
$$


Proposition 4.3. The $\star$ - product (4.6) endows $\mathcal{A}[[\hbar]]$ with a structure of a $\mathbb{C}$ algebra, and has the property

$$
f_{1} \star f_{2}=f_{1} f_{2}+\hbar \sum_{i, j=1}^{n} h^{i \bar{j}} \bar{\partial}_{j} f_{1} \partial_{i} f_{2}+O\left(\hbar^{2}\right) .
$$

Moreover, the linear functional $\left.\operatorname{tr}: \mathcal{A}_{c}[[\hbar]] \rightarrow \mathbb{C}\left[\hbar^{-1}, \hbar\right]\right]$

$$
\operatorname{tr}(f)=\int_{\mathbb{C}^{n}} e_{\hbar}(z, \bar{z}) f(z, \bar{z}) d \mu_{\hbar}(z, \bar{z}),
$$

is cyclically invariant:

$$
\operatorname{tr}\left(f_{1} \star f_{2}\right)=\operatorname{tr}\left(f_{2} \star f_{1}\right) .
$$

Proof. The property $f \star 1=1 \star f=f$, for all $f \in \mathcal{A}$, follows from the definition. The strong form (4.7) of the correspondence principle follows from the computation of $e^{(1)}$, given in Remark 4.1. The proof of the trace property is the same as in proposition 3.10 .

Remark 4.4. Using the previous remark, it is easy to find the first two terms of the normalized $\star$ - product. They are expressed only through elements of the inverse matrix $H^{-1}=\left(h^{i \bar{j}}\right)$ — "polarized Poisson tensor", and are given by the following expression

$$
\begin{aligned}
f_{1} \star f_{2} & =f_{1} f_{2}+\hbar \sum_{i, j=1}^{n} h^{i \bar{j}} \bar{\partial}_{j} f_{1} \partial_{i} f_{2}+\frac{\hbar^{2}}{2} \sum_{i, j=1}^{n} \sum_{k, l=1}^{n}\left(h^{i \bar{j}} h^{k \bar{l}} \bar{\partial}_{j l}^{2} f_{1} \partial_{i k}^{2} f_{2}\right. \\
& +h^{i \bar{j}} \bar{\partial}_{l}\left(h^{k \bar{l}}\right) \bar{\partial}_{j} f_{1} \partial_{i k}^{2} f_{2}+\partial_{i}\left(h^{i \bar{j}}\right) h^{k \bar{l}} \bar{\partial}_{j l}^{2} f_{1} \partial_{k} f_{2} \\
& \left.+\partial_{i}\left(h^{i \bar{j}}\right) \bar{\partial}_{l}\left(h^{k \bar{l}}\right) \bar{\partial}_{j} f_{1} \partial_{k} f_{2}\right)+O\left(\hbar^{3}\right) .
\end{aligned}
$$

Remark 4.5. Though the first two terms in $\star$ - product (4.6) are expressed through the Poisson tensor only, this is no longer true (without further restrictions on the Kähler metric) for the higher order terms. Therefore, the $\star$ - product (4.6) is not suitable for the deformation quantization of a complex Poisson structure on $\mathbb{C}^{n}$ - the problem solved by Kontsevich for arbitrary Poisson structures on $\mathbb{R}^{n}$ 21. Still, we believe that our approach can be successfully applied to the problem of deformation quantization of arbitrary complex Poisson structure on $\mathbb{C}^{n}$, if it is modified in the spirit of the supersymmetric approach to the Duistermaat-Heckman localization formula (see, i.e., 28]). Specifically, consider representation (3.1) for the non-normalized - - product in the remark 3.9, and notice that the "action" $S=\phi(z, \bar{z} ; v, \bar{v}) / \hbar+(H \xi, \xi)$ is not invariant under the supersymmetry transformation. Introduction of the unit into the normalized $\star$ - product (4.6) modifies the action in a way that cancels "vacuum diagrams", but still it does not restore the supersymmetry. We conjecture that the supersymmetric action can be obtained by an appropriate deformation mod $\hbar$ of the Kähler metric. Then, according to the paradigm of the supersymmetric quantum field theory, the corresponding Feynman diagrams will contain no loops and will depend only on the Poisson tensor and its derivatives. 
4.2. Formal Bergman kernel. Here we will show that the unit element $e_{\hbar}(z, \bar{z})$ can be interpreted as the kernel of a projection operator. Specifically, for any $f \in \mathcal{A}$ define the following formal integral

$$
P_{\hbar}(f)(z)=\oint_{\mathbb{C}^{n}} e_{\hbar}(z, \bar{v}) f(v, \bar{v}) e^{(\Phi(z, \bar{v})-\Phi(v, \bar{v})) / \hbar} d \mu_{\hbar}(v, \bar{v})
$$

as the formal asymptotic expansion around the point $v=z$ obtained by repeated "integration by parts". Namely, applying Stokes' formula $n$ times, reduce the integration of $(n, n)$-form

$$
e^{(\Phi(z, \bar{v})-\Phi(v, \bar{v}) / \hbar} d v^{1} \wedge \ldots \wedge d v^{n} \wedge d \bar{v}^{1} \wedge \ldots \wedge d \bar{v}^{n}
$$

over the $2 n$-chain - direct product of $n$ annuli centered at $z^{i}$ to the integral of $(n, 0)$ form over the $n$-cycle - direct product of $n$ circles of radii $\epsilon$ around $z^{i}, i=1, \ldots, n$, and iterate this procedure. The term-by-term evaluation of the limit $\epsilon \rightarrow 0$ results in a power series expansion in $\hbar$ (see Appendix for more details). Equivalently, the formal integral $\oint$ can be defined as the asymptotic of the distribution $\exp (\Phi(z, \bar{v})-$ $\Phi(v, \bar{v})) / \hbar)$ as $\hbar \rightarrow 0$.

This procedure is well-defined, since

$$
\Phi(z, \bar{v})-\Phi(v, \bar{v})=\sum_{i=1}^{n} \partial_{i} \Phi(z, \bar{v})\left(z^{i}-v^{i}\right)+\text { higher order terms }
$$

and the matrix $\partial_{i} \bar{\partial}_{j} \Phi(z, \bar{z})=h_{i \bar{j}}(z, \bar{z})$ is non-degenerate. Coefficients of this power series expansion in $\hbar$ are given by infinite sums, convergent only when function $f$ and Kähler potential $\Phi$ are real-analytic. In the $C^{\infty}$-category, these coefficients should be interpreted as infinite jets. By $\mathbb{C}[[\hbar]]$-linearity, $P_{\hbar}$ extends to the linear mapping $P_{\hbar}: \mathcal{A}[[\hbar]] \rightarrow \mathcal{J}[[\hbar]]$, where $\mathcal{J}$, where $\mathcal{J}$ is the space of global sections of the bundle of formal (with respect to $\hbar$ ) infinite jets over $\mathbb{C}^{n}$.

Proposition 4.6. The mapping $P_{\hbar}$ takes values $\mathcal{J}_{\text {hol }}$ - the space of global sections of of the bundle of formal holomorphic jets over $\mathbb{C}^{n}$.

Proof. It is clear that in the real-analytic category the integral (4.8), when it converges, is a holomorphic function. For the asymptotics of the integral, this is an algebraic property of coefficients. Therefore, the same is true for the formal power series with the coefficients in infinite jets in the $C^{\infty}$-category.

Proposition 4.7. Operator $P_{\hbar}$ is a projector, $P_{\hbar}^{2}=P_{\hbar}$.

Proof. This follows from the identity between two types of formal integrals

$$
\int_{\mathbb{C}^{n}} f(v, \bar{v}) e^{\phi(z, \bar{z} ; v, \bar{v}) / \hbar} d \mu_{\hbar}(v, \bar{v})=\oint_{\mathbb{C}^{n}} \tilde{f}_{z}(v, \bar{v}) e^{(\Phi(z, \bar{v})-\Phi(v, \bar{v})) / \hbar} d \mu_{\hbar}(v, \bar{v}),
$$

where $\tilde{f}_{z}(v, \bar{v})=f(v, \bar{v}) e^{(\Phi(v, \bar{z})-\Phi(z, \bar{z})) / \hbar}$, proved in Appendix. Namely, for $f \in \mathcal{F}$ and $z \in \mathbb{C}^{n}$ set $g_{z}(v)=f(v) e^{-(\Phi(v, \bar{z})-\Phi(z, \bar{z}) / \hbar}$. Using the property of the unit and that $g_{z}(v)$ is holomorphic in $v$, we get

$$
\begin{aligned}
f(z) & =g_{z}(z)=\left(e_{\hbar} \bullet g_{z}\right)(z, \bar{z}) \\
& =\int_{\mathbb{C}^{n}} e_{\hbar}(z, \bar{v}) g_{z}(v) e^{\phi(z, \bar{z} ; v, \bar{v})) / \hbar} d \mu_{\hbar}(v, \bar{v}) \\
& =\oint_{\mathbb{C}^{n}} e_{\hbar}(z, \bar{v}) f(v) e^{(\Phi(z, \bar{v})-\Phi(v, \bar{v})) / \hbar} d \mu_{\hbar}(v, \bar{v}) \\
& =P_{\hbar}(f)(z) .
\end{aligned}
$$


4.3. Contravariant quantization. Here we define the formal analog of the mapping $I_{\hbar}$ from contravariant to covariant symbols as the following formal integral

$$
I_{\hbar}(\hat{f})(z, \bar{z})=\int_{\mathbb{C}^{n}} \hat{f}(v, \bar{v}) \frac{e_{\hbar}(z, \bar{v}) e_{\hbar}(v, \bar{z})}{e_{\hbar}(z, \bar{z})} e^{\phi(z, \bar{z} ; v, \bar{v}) / \hbar} d \mu_{\hbar}(v, \bar{v}) .
$$

By $\mathbb{C}[[\hbar]]$-linearity, it extends to the linear operator $I_{\hbar}: \mathcal{A}[[\hbar]] \rightarrow \mathcal{A}[[\hbar]]$ with the property

$$
I_{\hbar}=\mathrm{id}+\sum_{i=1}^{\infty} \hbar^{n} I^{(n)}
$$

where $I^{(n)}: \mathcal{A} \rightarrow \mathcal{A}$. Therefore, the operator $I_{\hbar}$ is invertible.

Define the $\star$-product of contravariant symbols as in (2.15),

$$
\hat{f}_{1} \hat{\star} \hat{f}_{2}=I_{\hbar}^{-1}\left(I_{\hbar}\left(\hat{f}_{1}\right) \star I_{\hbar}\left(\hat{f}_{2}\right)\right),
$$

which, of course, is equivalent to the $\star$-product of covariant symbols.

Define a formal Berezin-Toeplitz operator $F: \mathcal{J}_{\text {hol }}[[\hbar]] \rightarrow \mathcal{J}_{\text {hol }}[[\hbar]]$ with contravariant symbol $\hat{f} \in \mathcal{A}[[\hbar]]$ as follows: $F(g)=P_{\hbar}(\hat{f} g)$ for all $g \in \mathcal{J}_{\text {hol }}[[\hbar]]$. We have the following statement.

Proposition 4.8. The product $F=F_{1} F_{2}$ of formal Berezin-Toeplitz operators $F_{1}$ and $F_{2}$ with contravariant symbols $\hat{f}_{1}$ and $\hat{f}_{2} \in \mathcal{A}[[\hbar]]$ is a formal Berezin-Toeplitz operator with contravariant symbol $\hat{f}=\hat{f}_{1} \hat{\star} \hat{f}_{2}$.

Proof. This is a formal analog of lemma 2.2. Using Fubini theorem for formal integrals and the equality of two types of formal integrals proved in the Appendix, we have

$$
P_{\hbar}(\hat{f} g)(z)=\oint_{\mathbb{C}^{n}} e_{\hbar}(z, \bar{v}) f(z, \bar{v}) g(v) e^{(\Phi(v, \bar{z})-\Phi(v, \bar{v})) / \hbar} d \mu_{\hbar}(v, \bar{v}),
$$

where $f=I_{\hbar}(\hat{f})$. The same arguments also show that

$$
\left(F_{1} F_{2}\right)(g)(z)=\oint_{\mathbb{C}^{n}} e_{\hbar}(z, \bar{v})\left(f_{1} \star f_{2}\right)(z, \bar{v}) g(v) e^{(\Phi(z, \bar{v})-\Phi(v, \bar{v})) / \hbar} d \mu_{\hbar}(v, \bar{v}),
$$

and the statement follows.

Remark 4.9. Some interesting explicit formulas for the asymptotic expansion of the Bergman kernel on $\mathbb{C}$, as well as for the coefficients of the product $\hat{\star}$, were derived in [29]. It is also interesting that in [29] the deformation quantization appeared as a part of M-theory in the string theory.

Acknowledgments. For many inspiring discussions we are greatly indebted to M. Flato, who passed away in November 1998. We also would like to thank M. Kontsevich, S. Shatashvili and D. Sternheimer for helpful discussions, W. Taylor for bringing [29] to our attention, and A. Kogan for the help with the figures. The work of N.R. and L.T. was partially supported by the NSF grants DMS-97-09594 and DMS-95-00557 respectively. 


\section{APPENDIX}

Here we will prove the following statement.

Proposition 4.10. (Equality of two types of formal integrals)

$$
\int_{\mathbb{C}^{n}} f(v, \bar{v}) e^{\phi(z, \bar{z} ; v, \bar{v}) / \hbar} d \mu_{\hbar}(v, \bar{v})=\oint_{\mathbb{C}^{n}} \tilde{f}_{z}(v, \bar{v}) e^{(\Phi(z, \bar{v})-\Phi(v, \bar{v})) / \hbar} d \mu_{\hbar}(v, \bar{v}),
$$

where $\phi(z, \bar{z} ; v, \bar{v})=\Phi(z, \bar{v})+\Phi(v, \bar{z})-\Phi(z, \bar{z})-\Phi(v, \bar{v})$ is the Calabi function of the Kähler form and

$$
\tilde{f}_{z}(v, \bar{v})=f(v, \bar{v}) e^{(\Phi(v, \bar{z})-\Phi(z, \bar{z})) / \hbar} .
$$

Here the formal integral in the left hand side is understood as the formal Laplace expansion around the critical point $v=z$ of the exponential factor, i.e., as the asymptotic of the distribution $\exp (\phi(z, \bar{z} ; v, \bar{v}) / \hbar)$ as $\hbar \rightarrow 0$. The formal integral in the right hand side is understood as the asymptotic of the distribution $\exp ((\Phi(z, \bar{v})-$ $\Phi(v, \bar{v})) / \hbar)$ as $\hbar \rightarrow 0$.

Proof. We will start with case $n=1$; the precise definition of the formal integral $\oint$ will become clear in a due course. First, assume that $f \in C^{\infty}(\mathbb{C})_{\text {pol }}$, that the Calabi function has a single critical point at $v=z$, and, assuming that the integral

$$
J(f)(\hbar ; z, \bar{z})=\frac{\sqrt{-1}}{2 \pi \hbar} \int_{\mathbb{C}} f(v, \bar{v}) e^{\phi(z, \bar{z} ; v, \bar{v}) / \hbar} \partial_{v \bar{v}}^{2} \Phi(v, \bar{v}) d v \wedge d \bar{v}
$$

exists for $\hbar>0$, consider its the asymptotic behavior as $\hbar \rightarrow 0$. Replacement of the domain of integration by a neighborhood $U_{\delta}=\{v|v \in \mathbb{C}| v-z \mid,<\delta\}$ of $z \in \mathbb{C}$ (with certain $\delta>0$ depending on $f$ and $\Phi$ in the support of $f$ ) will result in an exponentially small error term as $\hbar \rightarrow 0$. (It would be also sufficient to consider terms that decay faster than any power $\hbar^{N}$ with $N>0$ ). Therefore,

$$
\begin{aligned}
J(f)(\hbar ; z, \bar{z}) & \cong \lim _{\epsilon \rightarrow 0} \frac{\sqrt{-1}}{2 \pi \hbar} \int_{\epsilon<|v-z|<\delta} f(v, \bar{v}) e^{\phi(z, \bar{z} ; v, \bar{v}) / \hbar} \partial_{v \bar{v}}^{2} \Phi(v, \bar{v}) d v \wedge d \bar{v} \\
& \cong \lim _{\epsilon \rightarrow 0} \frac{\sqrt{-1}}{2 \pi \hbar} \int_{\epsilon<|v-z|<\delta} \tilde{f}_{z}(v, \bar{v}) e^{(\Phi(z, \bar{v})-\Phi(v, \bar{v})) / \hbar} \partial_{v \bar{v}}^{2} \Phi(v, \bar{v}) d v \wedge d \bar{v}
\end{aligned}
$$

where the symbol $\cong$ stands for equality modulo exponentially small terms as $\hbar \rightarrow 0$.

Next, for fixed $z$, consider the following representation (cf [29])

$$
e^{(\Phi(z, \bar{v})-\Phi(v, \bar{v})) / \hbar} d v \wedge d \bar{v}=d\left(A(\hbar ; v, \bar{v}) e^{(\Phi(z, \bar{v})-\Phi(v, \bar{v})) / \hbar} d v\right),
$$

where

$$
A(\hbar ; v, \bar{v})=\sum_{n=1}^{\infty} A_{n}(v, \bar{v}) \hbar^{n}
$$

and satisfies the equation

$$
-\bar{\partial}_{v} A(\hbar ; v, \bar{v})+\frac{1}{\hbar} A(\hbar ; v, \bar{v})\left(\bar{\partial}_{v} \Phi(z, \bar{v})-\bar{\partial}_{v} \Phi(v, \bar{v})\right)=1,
$$

so that

$$
A_{1}(v, \bar{v})=\left(\bar{\partial}_{v} \Phi(z, \bar{v})-\bar{\partial}_{v} \Phi(v, \bar{v})\right)^{-1} \text { and } A_{n+1}(v, \bar{v})=A_{1}(v, \bar{v}) \bar{\partial}_{v} A_{n}(v, \bar{v}) .
$$


Applying Stokes' formula, we get

$$
\begin{gathered}
J(f)(\hbar ; z, \bar{z}) \cong \frac{\sqrt{-1}}{2 \pi \hbar} \lim _{\epsilon \rightarrow 0}\left\{\int_{C_{\epsilon}} \tilde{f}_{z}(v, \bar{v}) A(\hbar ; v, \bar{v}) \partial_{v \bar{v}}^{2} \Phi(v, \bar{v}) d v-\int_{\epsilon<|v-z|<\delta}\right. \\
\left.\left.A(\hbar ; v, \bar{v}) \partial_{\bar{v}}\left(\tilde{f}_{z} \partial_{v} \bar{\partial}_{v} \Phi\right)(v, \bar{v})\right) e^{(\Phi(z, \bar{v})-\Phi(v, \bar{v})) / \hbar} d v \wedge d \bar{v}\right\},
\end{gathered}
$$

where $C_{\epsilon}=\{v|v \in \mathbb{C}| v-z \mid,=\epsilon\}$, and we have used that the integral over the circle of radius $\delta$ around $z$ is exponentially small as $\hbar \rightarrow 0$.

Repeating this procedure, we obtain

$$
\begin{gathered}
\left.J(f)(\hbar ; z, \bar{z}) \cong \frac{\sqrt{-1}}{2 \pi \hbar} \lim _{\epsilon \rightarrow 0} \sum_{n=1}^{\infty}(-1)^{n} \int_{C_{\epsilon}} \mathcal{D}^{n}\left(\tilde{f}_{z} \partial_{v \bar{v}}^{2} \Phi\right)\right)(v, \bar{v}) A(\hbar ; v, \bar{v}) \\
e^{(\Phi(z, \bar{v})-\Phi(v, \bar{v})) / \hbar} d v,
\end{gathered}
$$

where $\mathcal{D}=A(\hbar, v, \bar{v}) \bar{\partial}_{v}$. Finally, expanding $A(\hbar ; v, \bar{v})$ into power series in $\hbar$ with coefficients $A_{n}(v, \bar{v})$ that have singularities $a_{n}(z, \bar{z})(v-z)^{-n}$ as $v \rightarrow z$, and using the generalized Cauchy formula

$$
\lim _{\epsilon \rightarrow 0} \frac{1}{2 \pi \sqrt{-1}} \int_{C_{\epsilon}} \frac{g(v, \bar{v})}{(v-z)^{n}} d v=\frac{\left(\partial_{z}\right)^{n-1} g(z, \bar{z})}{(n-1) !}, n=1,2, \ldots,
$$

which is valid for any $g \in C^{\infty}\left(U_{\delta}\right)$, we get an asymptotic expansion for $J(f)(\hbar ; z, \bar{z})$ in terms of a power series in $\hbar$. Clearly, the coefficients of this expansion are given by the infinite sums, convergent when the function $f$ and Kähler potential $\Phi$ are real-analytic. This expansion, extended to the formal category (with respect to $\hbar)$ with coefficients given by the global sections of the bundle of infinite jets over $\mathbb{C}$, is the definition of a formal integral $\oint$. Now proposition follows by the same arguments as in the proof of lemma 3.4 .

The proof for the case $n>1$ is similar and is left to the reader.

\section{REFERENCES}

[1] H. Weyl, Gruppentheorie und Quantenmechanik. Hirzel, Leipzig, 1928.

[2] E. Wigner, Phys. Rev., 40 (1932), 749. Math. Ann., 104 (1931), 570-578.

[3] J.E. Moyal, Quantum Mechanics as a Statistical Theory. Proc. Cambridge Phil. Soc., 45 (1949), 99-124.

[4] A. Groenewold, On the principles of elementary quantum mechanics. Physica, 12 (1946), 405-460.

[5] F.A. Berezin, Quantization. Math. USSR Izvestija, 8 (1974), 1109-1165.

[6] J. Rawnsley, M. Cahen and S. Gutt, Quantization on Kähler manifolds I. Geometric interpretation of Berezin's quantization. J. Geom. Phys., 7 (1990), 45-62.

[7] M. Cahen, S. Gutt and J. Rawnsley, Quantization of Kähler manifolds. II. Trans. Amer. Math. Soc., 337 (1993), 73-98.

[8] A. Karabegov, Deformation quantizations with separation of variables on a Kähler manifold. Commun. Math. Phys., 180 (1996), 745-755.

[9] F. Bayen, M. Flato, C. Fronsdal, A. Lichnerowicz, D. Sternheimer, Deformation theory and quantization. I. Deformation of symplectic structures. Ann. Phys., 111 (1978), 61-110.

[10] F. Bayen, M. Flato, C. Fronsdal, A. Lichnerowicz, D. Sternheimer, Deformation theory and quantization. II. Physical applications. Ann. Phys., 111 (1978), 111-151.

[11] J. Vey, Déformation du crochet de Poisson sur une variété symplectique. Comment. Math. Helv., 50 (1975) 421-454.

[12] M. Gerstenhaber, On the deformation of rings and algebras. Ann. Math., 79 (1964), 59-103.

[13] O.M. Neroslavsky et A.T. Vlasov, Sur le déformations de l'algè bre des fonctions d'une variété symplectique. C. R. Acad. Sc. Paris 292 I (1981), 71-76.

[14] A. Lichnerowicz, Existence and equivalence of twisted products on a symplectic manifold. Lett. Math. Phys., 3 (1979), 495-502. 
[15] M. De Wilde, P.B.A. Lecomte, Existence of the star-products and of formal deformations in Poisson-Lie algebra of arbitrary symplectic manifolds. Lett. Math. Phys., 7 (1983), 487-496.

[16] M. De Wilde, P.B.A. Lecomte, Formal deformations of the Poisson Lie algebra of a symplectic manifold and star-products. Existence, equivalence, derivations. In: Deformation Theory of Algebras and Structures and Applications, 897-960; Eds. M. Hazewinkel and M. Gerstenhaber, Kluwer Publishers, Dordrecht-Boston-London, 1988.

[17] H. Omori, Y. Maeda and A. Yoshioka, Weyl manifolds and deformation quantization. Adv. in Math., 85 (1991), 225-255.

[18] H. Omori, Y. Maeda and A. Yoshioka, Existence of a closed star product. Lett. Math.Phys., 26 (1992), 285-294.

[19] B. Fedosov, A simple geometric construction of deformation quantization. J. Diff. Geom., 40 (1994), 213-238.

[20] P. Etingof and D. Kazhdan, Quantization of Lie bialgebras. I. Selecta Math., New Series, 2 (1996), 1-41.

[21] M. Kontsevich, Deformation quantization of Poisson manifolds. I. preprint q-alg/9709040

[22] A.S. Cattaneo and G. Felder. A path integral approach to the Kontsevich quantization formula. preprint math.QA/9902090.

[23] D. Kazhdan, Lectures on QFT. IAS, Fall 1997.

[24] A. Karabegov, Pseudo-Kähler quantization on flag manifolds. preprint dg-ga/9709015.

[25] F.A. Berezin, Covariant and contravariant symbols of operators. Math. USSR Izvestija, 6 (1972), 1117-1151.

[26] M. Gerstenhaber and S.D. Shack, Deformation of algebras. In: Deformation Theory of Algebras and Structures and Applications, 11-264; Eds. M. Hazewinkel and M. Gerstenhaber, Kluwer Publishers, Dordrecht-Boston-London, 1988.

[27] A. Connes, M. Flato and D. Sternheimer, Closed star products and cyclic cohomology. Lett. Math. Phys., 24 (1992), 1-12.

[28] A. Schwarz and O. Zaboronsky, Supersymmetry and localization. Commun. Math. Phys., 183 (1997), 463-476.

[29] L. Cornalba and W. Taylor IV, Holomorphic curves from matrices. preprint hep-th/9807060.

Department of Mathematics, University of California at Berkeley, Berkeley, CA 94720, USA

E-mail address: reshetik@math.berkeley.edu

Department of Mathematics, SUny at stony Brook, Stony Brook, Ny 11794-3651, USA

E-mail address: leontak@math.sunysb.edu 